\title{
UCRL-CONF-207735
}

LAW RENCE LIVERMORE N A T IO N A L LABORATORY

\section{Raman spectroscopy under extreme conditions}

A. F. Goncharov, J. C. Crowhurst

November 5, 2004

5th Conference on Cryocrystals and Quantum Crystals Wroclaw, Poland

August 29, 2004 through September 4, 2004 
This document was prepared as an account of work sponsored by an agency of the United States Government. Neither the United States Government nor the University of California nor any of their employees, makes any warranty, express or implied, or assumes any legal liability or responsibility for the accuracy, completeness, or usefulness of any information, apparatus, product, or process disclosed, or represents that its use would not infringe privately owned rights. Reference herein to any specific commercial product, process, or service by trade name, trademark, manufacturer, or otherwise, does not necessarily constitute or imply its endorsement, recommendation, or favoring by the United States Government or the University of California. The views and opinions of authors expressed herein do not necessarily state or reflect those of the United States Government or the University of California, and shall not be used for advertising or product endorsement purposes. 


\title{
Raman spectroscopy under extreme conditions
}

\author{
Alexander F. Goncharov, Jonathan C. Crowhurst \\ Lawrence Livermore National Laboratory, University of California
}

We report the results of Raman measurements of various materials under simultaneous conditions of high temperature and high pressure in the diamond anvil cell (DAC). High temperatures are generated by laser heating or internal resistive (ohmic) heating or a combination of both. We present Raman spectra of cubic boron nitride (cBN) to $40 \mathrm{GPa}$ and up to $2300 \mathrm{~K}$ that show a continuous pressure and temperature shift of the frequency of the transverse optical mode. We have also obtained high-pressure Raman spectra from a new noble metal nitride, which we synthesized at approximately $50 \mathrm{GPa}$ and $2000 \mathrm{~K}$. We have obtained high-temperature spectra from pure nitrogen to $39 \mathrm{GPa}$ and up to 2000 $\mathrm{K}$, which show the presence of a hot band that has previously been observed in CARS measurements. These measurements have also allowed us to constrain the melting curve and to examine changes in the intramolecular potential with pressure.

\section{Introduction.}

The study of materials under extreme conditions is of fundamental scientific importance and is relevant to many diverse disciplines. A clear example is planetary science. The conditions of pressure and temperature that reign in the deep interior of the Earth and other planets can now be approached or even exceeded in the controlled environment of the laboratory. Data obtained in associated experiments are currently being used to refine our present conception of the physical and chemical nature of these planets.

Changing the pressure and temperature to which a material is exposed can of course bring about profound changes including phase transitions of many types and also chemical reactions. The effect of increasing pressure specifically is usually to drive the atoms of the material into more closely packed structures with a concomitant increase in the delocalization of valence electrons. Increasing the temperature tends to result in less ordered (higher entropy) structures, the disruption of chemical bonds and ionization. Insight into the nature of these mechanisms is also useful for practical applications. For 
example, information concerning the phase change in metastable solids can be used in order to evaluate whether the material with the properties of interest can be synthesized at ambient pressure [1].

For many decades shock-wave compression experiments were the sole way in which one could obtain experimental data at high pressure and temperatures [2]. The diagnostics include measurements of the equation of state, electrical conductivity, and even spectroscopy along the Hugoniot. The drawbacks of this technique are that it is difficult to vary temperature and pressure independently and the necessity to perform all the measurement in a very short period of time (less than few $\mu$ s). Laser heating experiments in a diamond anvil cell allow the possibility to study materials under extreme conditions (let us say up to $200 \mathrm{GPa}$ and $6000 \mathrm{~K}$ ), but in situ measurements are still extremely rare (see e.g., Ref. [3]).

Many optical spectroscopic techniques are however in principle ideally suited to probing materials under these conditions. Raman spectroscopy for example combines very well with the DAC and measurements can be made with rapidity on samples of maximum dimension of only several $\mu \mathrm{m}$. This latter ability is particularly useful for minimizing the problems associated with the enormous thermal gradients encountered in the DAC when samples are heated above approximately a thousand degrees (using for example laser heating which is highly localized). Large thermal backgrounds and difficulties in measuring pressure in situ at high temperature remain experimental problems to overcome, but time-resolved approaches appear to be promising. In this paper we focus on our Raman scattering technique under extreme conditions. To generate high temperatures, we have used two different methods: laser 
heating, and internal resistive heating. Our combined Raman/laser heating system effectively utilizes high-throughput dichroic optics to introduce the heating and Raman probe beams into the DAC collinear with the cell axis. We present Raman data obtained from $\mathrm{cBN}$ that provide a basis for using this material as an in situ pressure gauge at high temperature. Raman measurements in hot (to $2100 \mathrm{~K}$ ) nitrogen provide clear evidence of melting, and have allowed us to extend the melting curve of solid $\mathrm{N}_{2}$ to $48 \mathrm{GPa}$. The Raman spectra at high temperature show the presence of lower frequency vibron sidebands, which we have assigned to the vibrational transitions from excited energy levels ("hot bands") that were previously observed in shock-wave [4] and static [5] CARS experiments.

\section{Experimental details.}

\section{The internal resistively heated diamond anvil cell (IHDAC)}

This technique was pioneered by Liu and Basset [6]. Different versions of the same basic design utilized by other researchers [7-9], including the latest reported one, which uses a split gasket [9]. Conceptually, this device is very simple - a tiny wire or ribbon in the cell cavity is resistively heated. The ribbon is pressed directly into metal leads, which are used to apply modest potential difference. Necessary electrical insulation (from the metal gasket) and thermal insulation (from the diamonds) is achieved by a layer composed of alumina or zirconia and in (appropriate areas) a small quantity of epoxy. Before indenting the leads, the central part of the gasket is drilled out and refilled with pure alumina. Compared with laser heating, the IHDAC offers long-term temperature stability as well as much smaller temperature gradients (see [9] and Refs. therein). Once built, the 
IHDAC is also very simple to operate,
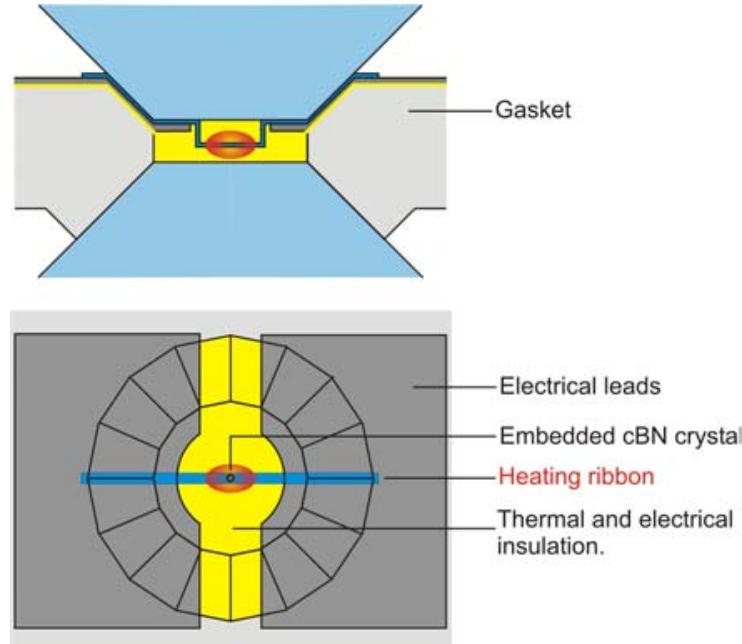

Fig. 1. Schematic of internally heated diamond anvil cell. and since it is so compact (requiring

only a modest power supply) it is

convenient for use in situations where

travel is required, or space is at a

premium (e.g., at synchrotrons). We

have tested an IHDAC design of our

own and have thus far achieved a

measured temperature of at least $3500 \mathrm{~K}$

at a pressure of $30 \mathrm{GPa}$. In the present

experiment a small sample of $\mathrm{cBN}(10-20 \mu \mathrm{m})$ was pressed into the heating wire to ensure efficient thermal contact. The heating ribbon could also be simultaneously laser heated, and this combination proved convenient in several instances.

\section{Laser heating of samples in the diamond anvil cell (LHDAC)}

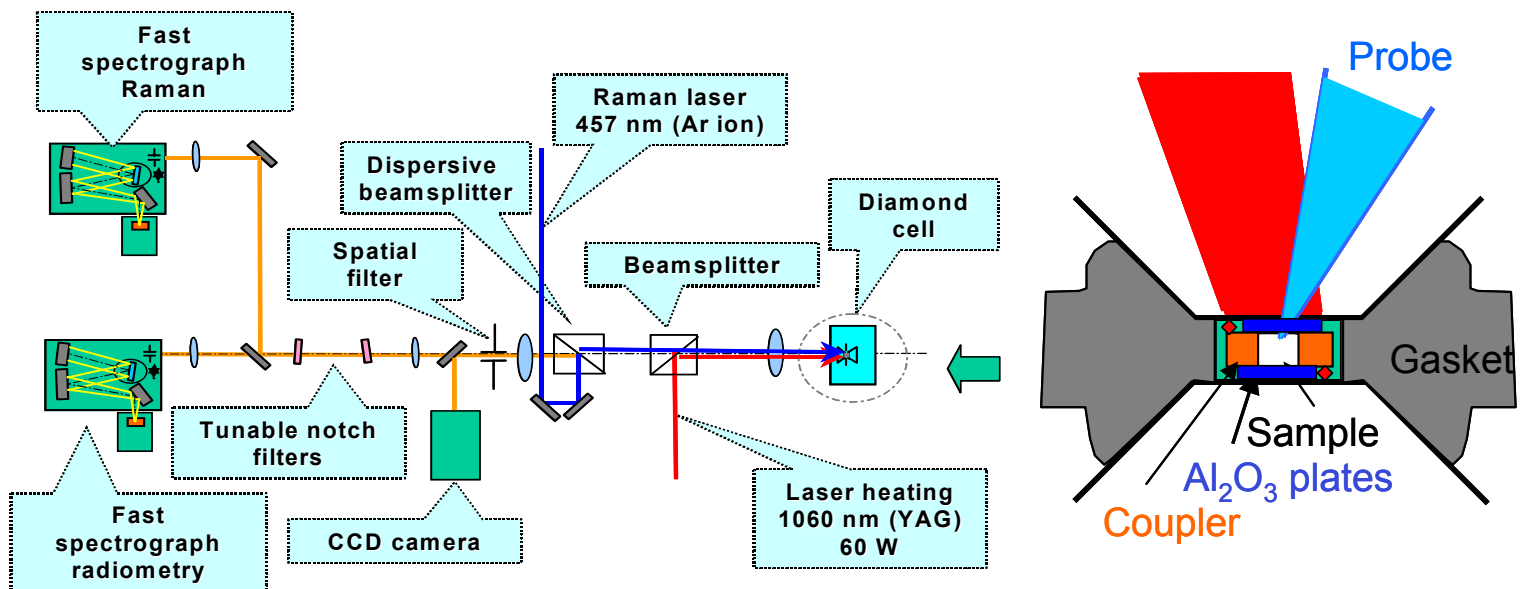

Fig. 2. Schematic of our laser heating optical setup. Right panel: arrangement of sample in a diamond anvil cell cavity. 
Our laser heating apparatus (Fig. 2) combines conventional one-sided laser heating with a Raman system. A Mitutoyo near IR 20x long-working distance lens was employed for the collection of Raman spectra in the backscattering geometry and for focusing of the heating IR radiation on the sample using the same optical path. A continuous-wave $50 \mathrm{~W}$ Nd:YAG laser was used as the heating source. In order to match the entrance puple of the objective lens and to provide more uniform temperature distribution at the sample, we used a custom-made beam contractor. An unpolarized output beam of the laser was split into two beams with orthogonal polarizations using a polarizing beam-splitter cube. The YAG laser radiation was injected into the Raman system using two polarizing beam-splitter cubes (for each of the beams with different polarizations; only one is shown on the Fig. 2 for simplicity) and was focused to a 20-30 $\mu \mathrm{m}$ spot. The heating laser power was increased in steps (by rotating a $\lambda / 2$ wave plate coupled to a polarization cube).

The Raman part of our confocal Raman system has previously been described in detail [10]. We used the $458 \mathrm{~nm}$ line of a $300 \mathrm{~mW}$ Ar ion laser to excite the Raman spectra. The Ar ion laser radiation was injected into the Raman system using a beamsplitter made of a Kaiser Optics laser bandpass filter. The scattered/emitted by sample radiation was analyzed by two dedicated single-stage grating spectrographs: Jobin Yvon HR460 for Raman measurements and Ocean Optics USB2000 - for thermal radiometry. We determined the temperature of the sample in IHDAD, LHDAC and combined experiments by measuring thermal emission spectra over a wide, continuous spectral region. The Planck radiation function is then fitted to the measured spectra. Since the heated region in the IHDAC is comparatively large, and the temperature gradients 
small, only a modest uncertainty in temperature determination was experienced for IHDAC ( $<30 \mathrm{~K})$, compared to that in LHDAC experiments (up to $100 \mathrm{~K}$ ). The radiometry method gives reliable results at temperatures above approximately $1000 \mathrm{~K}$, because at lower temperature the signal is too weak to provide adequate statistics. We also estimated temperature by analyzing the relative intensities of the Stokes and AntiStokes Raman peaks and also by measuring the relative intensity of hot vibrons band with respect to the fundamental (in case of $\mathrm{N}_{2}$, see below). This technique is based on an intrinsic property of the studied system, so it is presumably more accurate. The drawback

of this method is a high uncertainty in the case of a very weak anti-Stokes signal, and also in case when the temperature is large compared to the energy of the Raman transition being used. Where the temperature range was appropriate for the use of both techniques consistent results were obtained.

\section{Experimental results.}

\section{Cubic boron nitride}

In situ measurements of pressure at temperatures beyond $1000 \mathrm{~K}$ is a challenging task. Usually measurements are made at room temperature with a subsequent estimation of the thermal pressure [11], or in situ ruby (or other gauge) fluorescence measurements from the relatively cold part of the sample cavity [12]. Both these methods may have large uncertainties and ideally should be substituted by in situ measurements using a sensor positioned in the close vicinity of the sample. Only materials that are chemically inert are likely to be useful for this purpose. Here we present preliminary results obtained from of cBN to $40 \mathrm{GPa}$ at temperatures of up to $2300 \mathrm{~K}$ using our combined IHDAD and 
LHDAC techniques. The use of this material as a pressure gauge at high temperature requires an accurate calibration function, which is proposed to contain coupled pressure and temperature terms [13] based on measurements at lower temperature.

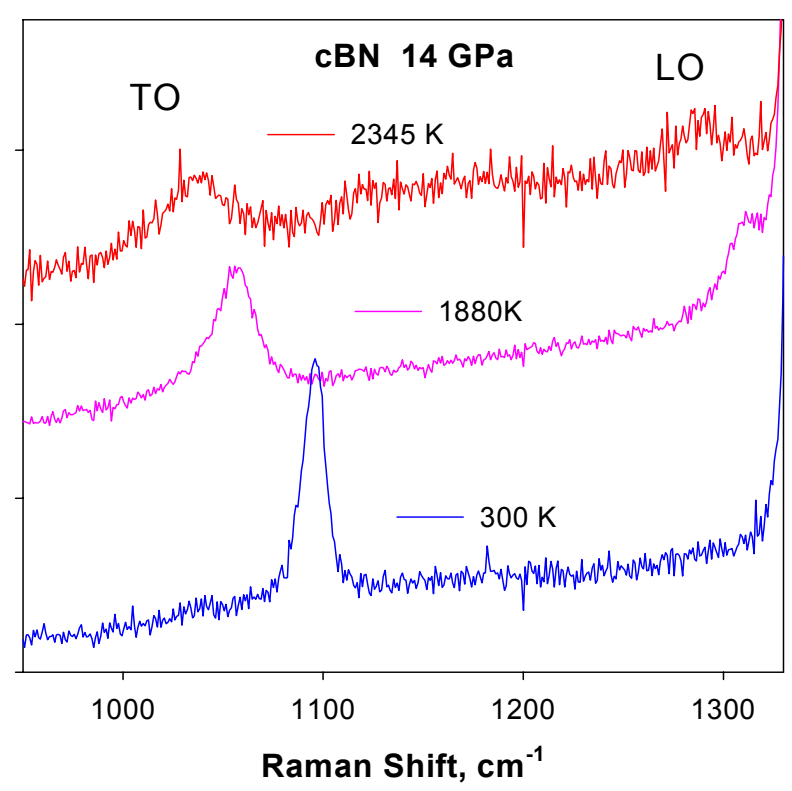

Fig. 3. Raman spectra of cBN up to $2345 \mathrm{~K}$ at $14 \mathrm{GPa}$.

Raman spectra of cBN at elevated temperatures (Fig. 3) show a decrease of frequency of the transverse (TO) and longitudinal (LO) optical phonons similar to that observed at ambient pressure. We analyzed only the behavior of the TO mode because (unlike the LO mode) the corresponding band is well separated from the peak due to Raman scattering in diamond over the entire range of experimental pressure and temperature. We performed two series of experiments: one in which alumina was used as the pressure medium and one, which used argon. The temperature shifts of the observed frequencies are substantially different. In the case of alumina the frequency shift is significantly smaller than that expected from extrapolation of lower temperature literature data [13]. We 
impute this fact to thermal pressure, which is generated during heating. Since alumina is a fairly rigid material, which can sustain large stresses [14], the hot area in the sample cavity was likely to be at a substantially larger pressure than the colder areas in which pressure was measured. We estimated pressure at high temperature using the results of Ref. [13]. In the second set of experiments (with an Ar transmitting medium), we find almost no thermal pressure when heating to approximately $2000 \mathrm{~K}$, which is consistent with the low shear strength of Ar at high temperature near the melting line [15]. Our experiments show that $\mathrm{cBN}$ can be conveniently used as in situ pressure sensor to at least $2400 \mathrm{~K}$. Moreover, our results provide a basis for the optical calibration of $\mathrm{cBN}$ as a pressure sensor. In future we will also perform x-ray diffraction measurements of the equation of state of $\mathrm{cBN}$ under these conditions and will compare all our results to first principles calculations.

\section{Synthesis of a noble metal nitride}

Transition metal nitrides are of great technological importance traditionally because of their strength and durability, but also because of other interesting physical properties (e.g. optical, electronic, and magnetic [16-21]). Recently, several experimental and theoretical investigations have been made into the synthesis and properties of nitrides produced under extreme conditions of pressure and temperature. Novel phases of known nitrides have been successfully synthesized [18-21] as well as at least one entirely new compound [21]. The latter work reports the synthesis of the binary noble metal nitride PtN. This material was found to be stable under ambient conditions and to possess a bulk modulus of $372 \mathrm{GPa}$, remarkable for being nearly $100 \mathrm{GPa}$ higher than the pure metal. 
We have now produced under extreme conditions a recoverable nitride of iridium. Raman scattering was used as the principle diagnostic in this investigation.

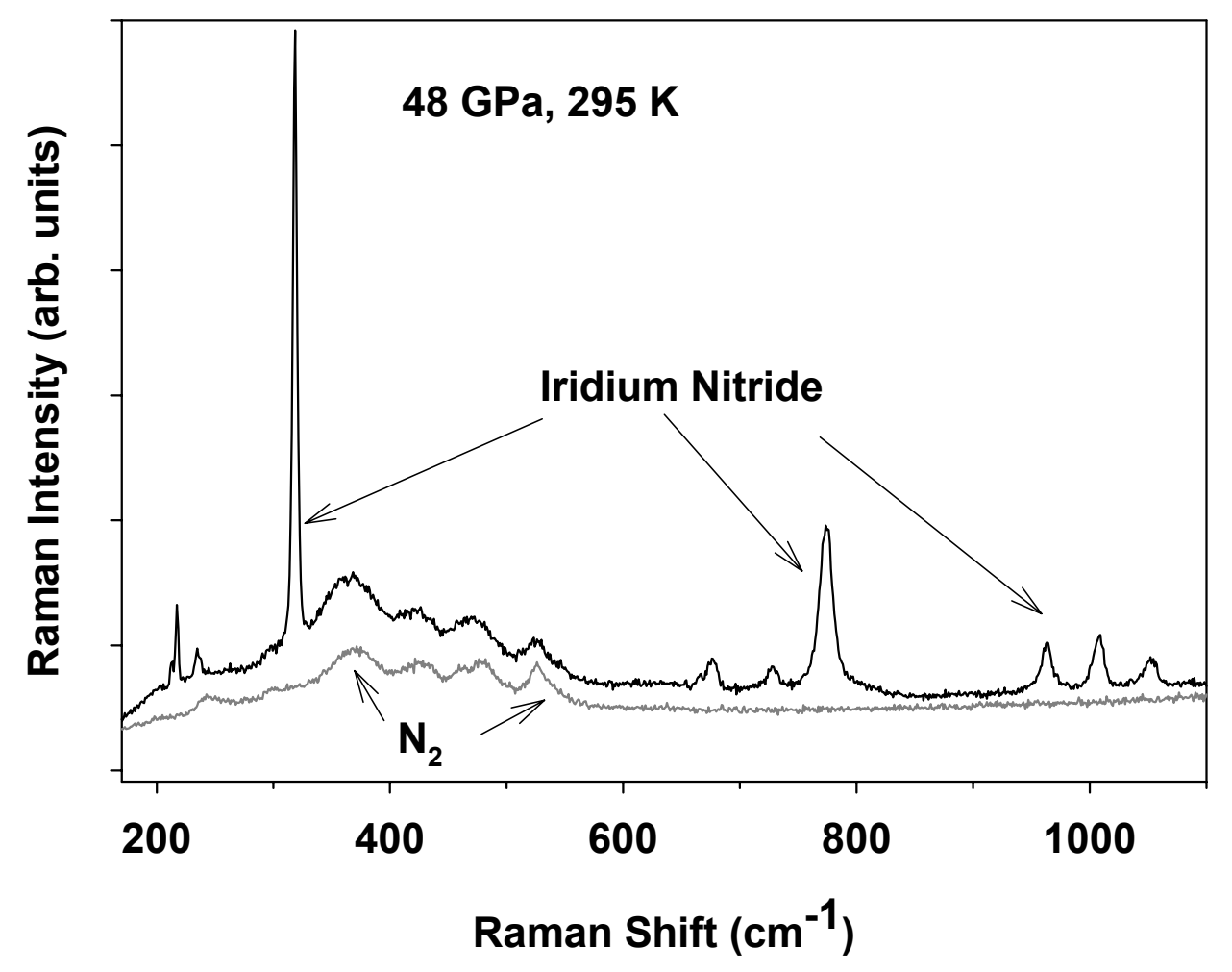

Fig. 4. Raman spectrum of a synthesized at high pressure and temperature and quenched to ambient temperature Iridium nitride in a DAC at $48 \mathrm{GPa}$ (upper trace). The lower trace is the Raman spectrum of nitrogen, shown for the comparison.

Samples were loaded in either the form of powder or single squares (of 30x10 microns) and laser heated in the presence of nitrogen. Above approximately $50 \mathrm{GPa}$ and $2000 \mathrm{~K}$ the Raman spectra exhibited sharp and intense modes indicative of crystalline material (Fig. 4). Microprobe analysis of the recovered material clearly demonstrated the strong presence of nitrogen. We are currently applying other diagnostics to the recovered sample including scanning electron microcopy and x-ray photo-electron spectroscopy. 
Nitrogen under extreme conditions

Nitrogen atoms form a diatomic molecule that is triply bonded. Thus, we may expect complex chemical behavior under extreme conditions of pressure and temperature related to destabilization of this bond. Theoretical calculations predict molecular dissociation below $50 \mathrm{GPa}$ [22]. Recently, several new phases of nitrogen have been reported in static experiments with external resistive heating $[23,24]$. These phases have been interpreted as extended (nonmolecular) and polyatomic (molecular) materials [2527]. Moreover, theoretically predicted [26] "cubic gauche" phase is reported in a laser heating study [28]. Shock-wave experiments [29,30] indicate molecular dissociation of $\mathrm{N}_{2}$ above $35 \mathrm{GPa}$ and metallization at $120 \mathrm{GPa}$.

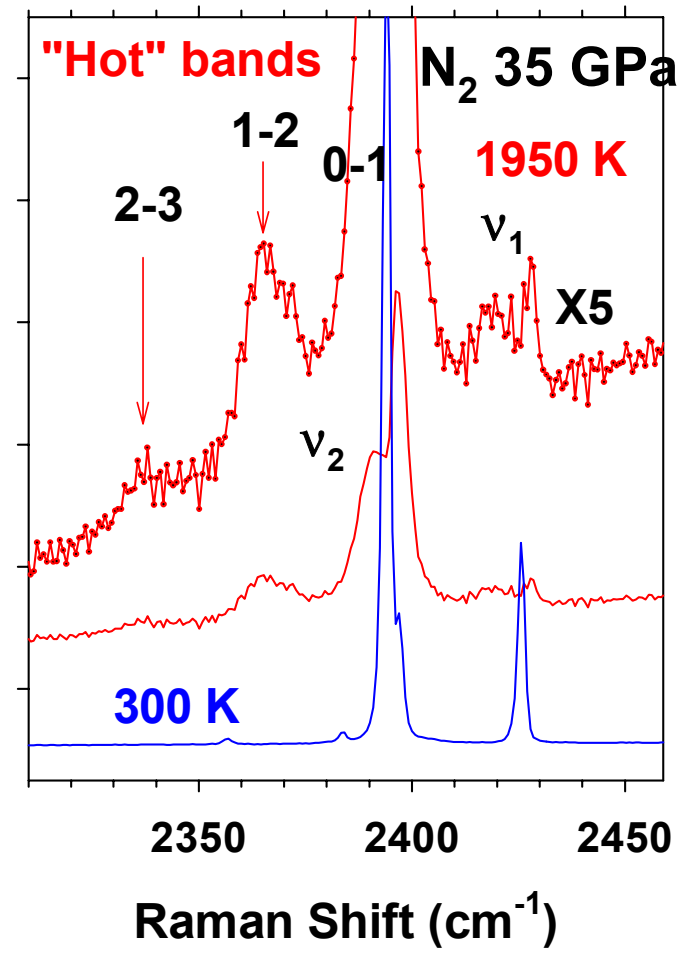

Fig. 5. Raman spectra of $\mathrm{N}_{2}$ up to $1950 \mathrm{~K}$ at $35 \mathrm{GPa}$. 


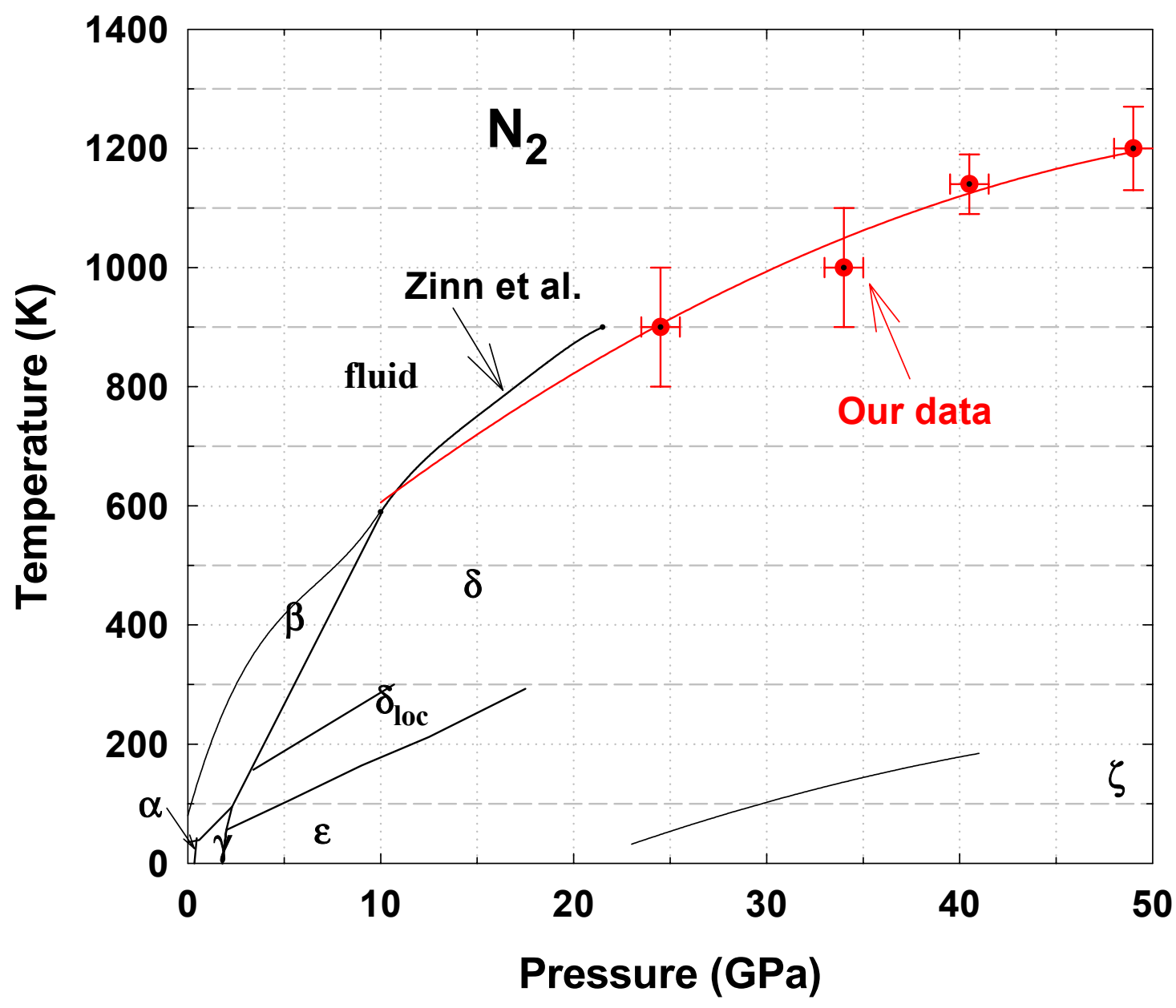

Fig. 6. Phase diagram of nitrogen.

Here we present here selected results of Raman measurements of $\mathrm{N}_{2}$ to $39 \mathrm{GPa}$ and $2000 \mathrm{~K}$ using laser heating. Raman spectra at high temperature reveal additional vibron bands at lower frequency than the main $v_{2}$ band (Fig. 4). We assign it to a transition from excited states, as was done previously in a coherent anti-Stokes Raman scattering experiment $[4,5]$. The pressure dependence of this band allows us to obtain information about the intra-molecular potential function, which is expected to become 
less steep when approaching the dissociation limit. We find that in the pressure range studied the spacings between the transitions $(0-1,1-2$, and 2-3) is constant and independent of pressure within the accuracy of our measurements.

Our experiments also allow determining the melting curve line of nitrogen (Fig. 6). At the melting, a new vibron corresponding to liquid state appears at slightly lower frequency comp.ared to the $v_{2}$ vibron (Fig. 5). Our data are in a good agreement with the results of Zinn et al., (Ref. [31]), who also used a Raman technique in combination with the externally heated DAC.

This work was performed under the auspices of the University of California under US DOE Contract No. W-7405-Eng-48 at LLNL.

Bibliography.

[1] V. V. Brazhkin, A. G. Lyapin, Nature Materials 3, 497 (2004).

[2] W. J. Nellis in R. J. Hemley and et al., eds., Proceedings of the International School of Physics "Enrico Fermi", Course CXLVII, Società Italiana di Fisica, (2002) p. 109.

[3] G. Shen, M. L. Rivers, Y. Wang, and S. R.Sutton, Rev. Sci. Instrum. 72, 1273 (2002)

[4] S. C. Schmidt, D. S. Moore, and M. S. Shaw, Phys. Rev. B 35, 493 (1987).

[5] B. J. Baer and C.-S. Yoo, Rev. Sci. Instrum. 75, 555 (2004)

[6] L.-G. Liu and W. A. Bassett, J. Geophys. Res. 80, 3777 (1975).

[7] H.-K Mao, P. M. Bell, and C. Hadidiacos, in: High-Pressure Research in Mineral Physics, edited by M. H. Manghnani and Y. Syono (Terra Scientific, Washington, DC, 1987) 135 (1987).

[8] R. Boehler, M. Nicol, C. S. Zha, and M. L. Johnson, Physica B \& C 139, 916 (1986).

[9] C.-S. Zha, and W. A. Bassett, Rev. Sci. Instrum, 74, 1255 (2003).

[10] A. F. Goncharov, V. V. Struzhkin, R. J. Hemley, H-K. Mao, Z. Liu. In Science and Technology of High Pressure, edited by M. H. Manghnani, W. J. Nellis, and M. F. Nicol (Universities Press, Hyderabad, India, 2000), pp. 95-99.

[11] A. Dewaele, G. Fiquet, and Ph. Gillet, Rev. Sci. Instrum. 69, 2421 (1998). 
[12] R. Boehler, Rev. Geophys., 38, 221 (2000).

[13] F. Datchi, and B. Canny, Phys. Rev. B. 69, 144106 (2004).

[14] C. Mead and R. Jeanloz, Phys. Rev. B. 42, 2532 (1990).

[15] R. Boehler, M. Ross, P. Söderlind, and D. B. Boercker, Phys. Rev. Lett. 86, 5731 (2001).

[16] S. T. Oyama, The Chemistry of Transition Metal Carbides and Nitrides; Blackie Academic \& Professional: London, U.K., 1996.

[17] H.O. Pierson, Handbook of Refractory Carbides and Nitrides: Properties, Characteristics, and Applications (Noyes Publications, Westwood, NJ, 1996).

[18] A. Zerr et al., Nature (London) 400, 340 (1999).

[19] K. Leinenweber, M. O'Keeffe, M.S. Somayazulu, H. Hubert, P.F. McMillan, and G.H. Wolf, Chem.-Eur. J. 5, 3076 (1999)

[20] A. Zerr, G. Miehe, and R. Riedel, Nature Materials 2, 185 (2003).

[21] E. Gregoryanz, C. Sanloup, M. Somayazulu, J. Badro, G. Fiquet, H-K Mao, R. J. Hemley, Nature Materials 3, 294 (2004).

[22] A. K. McMahan and R. LeSar, Phys. Rev. Lett. 54, 1929 (1985).

[23] A. F. Goncharov et al., Phys. Rev. Lett., 85, 1262 (2000).

[24] E. Gregoryanz et al., Phys. Rev. B 66, 224108 (2002).

[25] R. M. Martin and R. J. Needs, Phys. Rev. B 34, 5082 (1986).

[26] C. Mailhiot, L. H. Yang, and A. K. McMahan, Phys. Rev. B 46, 14419 (1992).

[27] R. J. Bartlett, Chemistry and Industry, 4, 140 (2000).

[28] M. I. Eremets, A. G. Gavriliuk, I. A. Trojan, D. A. Dzivenko, and R. Boehler, Nature Materials 3, 558 (2004)

[29] H. B. Radousky et al., Phys. Rev. Lett. 57, 2419 (1986).

[30] R. Chau et al., Phys. Rev. Lett. 90, 245501 (2000).

[31] S. Zinn, D. Schiferl, and M. F. Nicol, J. Chem. Phys. 87, 1267 (1987); S. C.

Schmidt, D. Schiferl, A. S. Zinn, D. D. Ragan, and D. S. Moore, J. Appl. Phys. 69, 2793 (1991). 